\title{
Effect of the Coulomb interaction on the liquid-gas phase transition of nuclear matter
}

\author{
Rana Nand周 and Stefan Schramm话 \\ Frankfurt Institute for Advanced Studies, \\ 60438 Frankfurt am Main, Germany
}

\begin{abstract}
We investigate the role of the Coulomb interaction on the liquid-gas phase transition of nuclear matter with three different values of proton fraction $\left(Y_{p}=0.5,0.3\right.$ and 0.1$)$, relevant for heavy-ion physics as well as various astrophysical scenarios, within the framework of quantum molecular dynamics. We perform simulations for a wide range of density and temperature with and without the Coulomb interaction and calculate the two-point correlation functions of nucleon density fluctuations for all the configurations to determine the phase transition region. We also determine the critical end point of the liquid-gas phase transition for all three values of proton fraction considered. We observe that the Coulomb interaction reduces the transition temperature by $\gtrsim 2 \mathrm{MeV}$ for nuclear matter with $Y_{p}=0.5$ and 0.3 and by $\sim 1 \mathrm{MeV}$ for nuclear matter with $Y_{p}=0.1$. However, the critical density is found to be more or less insensitive to the Coulomb interaction.
\end{abstract}

\footnotetext{
* nandi@fias.uni-frankfurt.de

$\dagger$ schramm@fias.uni-frankfurt.de
} 


\section{INTRODUCTION}

One main focus of heavy-ion collision experiments is to understand the properties of the liquid-gas phase transition in nuclear matter [1, 2]. This phase transition is also important for various astrophysical reasons. For example, it plays a significant role in the dynamics of supernova explosions [3 5] and neutron stars [5] 8 . There exist numerous studies on the liquid-gas phase-transition of both symmetric and asymmetric nuclear matter using non-relativistic Skyrme interactions [9-13] as well as relativistic mean-field models [4, 14] 16, 18]. Studies of the the liquid-gas mixed phase are mostly done using the Gibbs phase equilibrium conditions derived in bulk limit i.e. ignoring the finite-size effects due to the surface and Coulomb interactions [4, 13, 18]. Several authors have included finite-size effects but at different levels of approximations and obtained considerable effects on the liquidgas phase transition properties [9, 19, 22]. Recently, in Ref. [23] the influence of surface and Coulomb interactions on the liquid-gas phase transition of stellar matter is studied in a consistent manner by using a compressible liquid-drop model where the surface and Coulomb contributions are included while deriving the phase equilibrium conditions. They found that the finite-size effects significantly reduce the region of liquid-gas mixed phase and the critical temperature $\left(T_{c}\right)$ is much lower than that obtained with bulk calculation.

In this article, we investigate the influence of Coulomb interaction on the liquid-gas phase transition of nuclear matter with quantum molecular dynamics (QMD) simulation. In particular, we use the QMD model developed by Maruyama et al [24] and extensively used to study the various properties of pasta phases that appear at the liquid-gas transition region, in recent years [25 29].

\section{FORMALISM}

In the QMD approach the state of a nucleon is represented by a Gaussian wave packet (we set $\hbar=c=1$ ):

$$
\psi_{i}(\mathbf{r})=\frac{1}{\left(2 \pi C_{W}\right)^{3 / 4}} \exp \left[-\frac{\left(\mathbf{r}-\mathbf{R}_{\mathbf{i}}\right)^{2}}{4 C_{W}}+i \mathbf{r} \cdot \mathbf{P}_{\mathbf{i}}\right],
$$

where $\mathbf{R}_{i}$ and $\mathbf{P}_{i}$ denote the centers of the position and momentum of the wave packet

$i$, respectively, with the corresponding width $C_{W}$. Then the total wave function for the 
$\mathcal{N}$-nucleon system is obtained by taking the direct product of single-nucleon wave functions

$$
\Psi(\{\mathbf{r}\})=\prod_{i}^{\mathcal{N}} \psi_{i}(\mathbf{r})
$$

Here we adopt the QMD Hamiltonian developed by Maruyama et al. [24], to simulate the nuclear matter at sub-saturation densities. The Hamiltonian consists of several terms :

$$
\mathcal{H}=T+V_{\text {Pauli }}+V_{\text {Skyrme }}+V_{\text {sym }}+V_{\text {MD }}+V_{\text {Coul }},
$$

where $T$ is the kinetic energy, $V_{\text {Pauli }}$ is the Pauli potential, which phenomenologically incorporates the Pauli exclusion principle, $V_{\text {Skyrme }}$ represents the nucleon-nucleon potential similar to Skyrme-like interactions, $V_{\mathrm{sym}}$ is the isospin-dependent potential related to the symmetry energy, $V_{\mathrm{MD}}$ is the momentum-dependent potential included as Fock terms of Yukawa-type interactions and finally, $V_{\text {Coul }}$ is the Coulomb potential. The explicit expressions for all the terms are given as [24, 29]:

$$
\begin{aligned}
T & =\sum_{i} \frac{\mathbf{P}_{i}^{2}}{2 m_{i}}, \\
V_{\text {Pauli }} & =\frac{C_{\mathrm{P}}}{2}\left(\frac{1}{q_{0} p_{0}}\right)^{3} \sum_{i, j(\neq i)} \exp \left[-\frac{\left(\mathbf{R}_{i}-\mathbf{R}_{j}\right)^{2}}{2 q_{0}^{2}}-\frac{\left(\mathbf{P}_{i}-\mathbf{P}_{j}\right)^{2}}{2 p_{0}^{2}}\right] \delta_{\tau_{i} \tau_{j}} \delta_{\sigma_{i} \sigma_{j}}, \\
V_{\text {Skyrme }} & =\frac{\alpha}{2 \rho_{0}} \sum_{i, j(\neq i)} \rho_{i j}+\frac{\beta}{(1+\tau) \rho_{0}^{\tau}} \sum_{i}\left[\sum_{j \neq i)} \tilde{\rho}_{i j}\right]^{\tau}, \\
V_{\mathrm{sym}} & =\frac{C_{\mathrm{s}}}{2 \rho_{0}} \sum_{i, j(\neq i)}\left(1-2\left|\tau_{i}-\tau_{j}\right|\right) \rho_{i j} \\
V_{\mathrm{MD}} & =\frac{C_{\mathrm{ex}}^{(1)}}{2 \rho_{0}} \sum_{i, j(\neq i)} \frac{1}{1+\left[\frac{\mathbf{P}_{i}-\mathbf{P}_{j}}{\mu_{1}}\right]^{2}} \rho_{i j}+\frac{C_{\mathrm{ex}}^{(2)}}{2 \rho_{0}} \sum_{i, j(\neq i)} \frac{1}{1+\left[\frac{\mathbf{P}_{i}-\mathbf{P}_{j}}{\mu_{2}}\right]^{2}} \rho_{i j}, \\
V_{\mathrm{Coul}} & =\frac{e^{2}}{2} \sum_{i, j(\neq i)}\left(\tau_{i}+\frac{1}{2}\right)\left(\tau_{j}+\frac{1}{2}\right) \iint d^{3} \mathbf{r} d^{3} \mathbf{r}^{\prime} \frac{1}{\left|\mathbf{r}-\mathbf{r}^{\prime}\right|} \rho_{i}(\mathbf{r}) \rho_{j}\left(\mathbf{r}^{\prime}\right),
\end{aligned}
$$

where $\rho_{0}=0.165 \mathrm{fm}^{-3}$ is the normal nuclear matter density, $\sigma_{i}$ and $\tau_{i}(1 / 2$ for protons and $-1 / 2$ for neutrons) are the nucleon spin and isospin, respectively and $\rho_{i j}$ and $\tilde{\rho}_{i j}$ represent the overlap between single-nucleon densities and are defined as

$$
\rho_{i j} \equiv \int d^{3} \mathbf{r} \rho_{i}(\mathbf{r}) \rho_{j}(\mathbf{r}), \quad \tilde{\rho}_{i j} \equiv \int d^{3} \mathbf{r} \tilde{\rho}_{i}(\mathbf{r}) \tilde{\rho}_{j}(\mathbf{r}),
$$

whereas the single-nucleon densities are given by

$$
\rho_{i}(\mathbf{r})=\left|\psi_{i}(\mathbf{r})\right|^{2}=\frac{1}{\left(2 \pi C_{W}\right)^{3 / 2}} \exp \left[-\frac{\left(\mathbf{r}-\mathbf{R}_{i}\right)^{2}}{2 C_{W}}\right],
$$


TABLE I. Parameter set for the interaction [24]

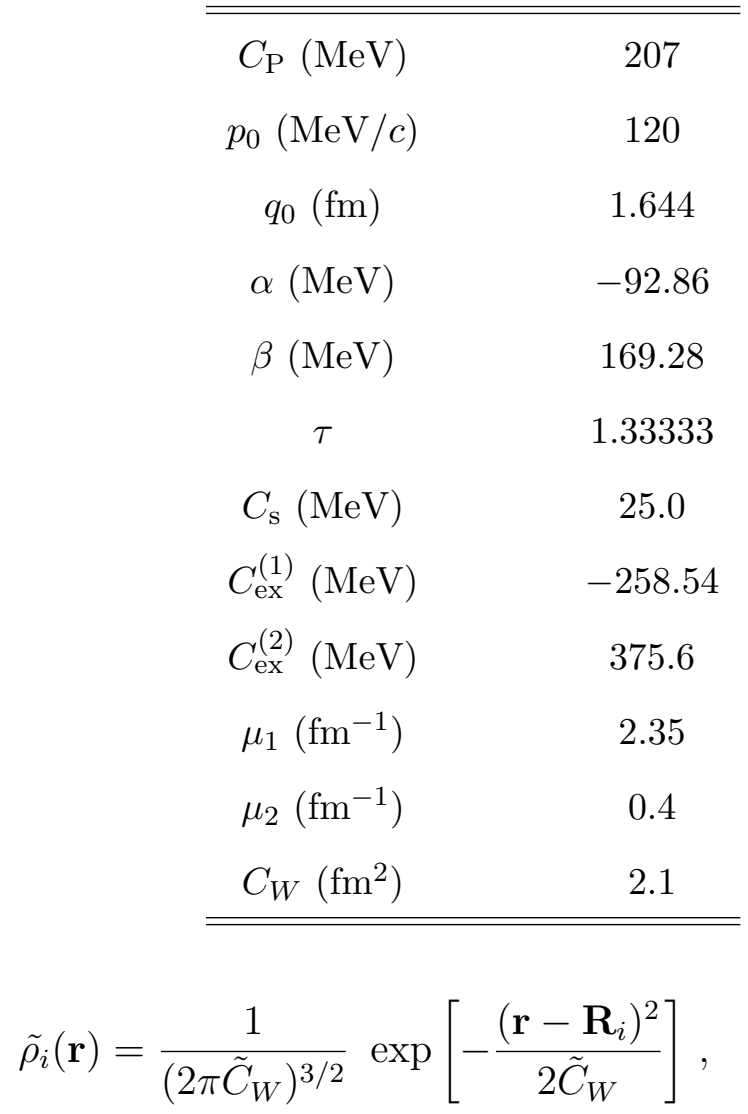

with

$$
\tilde{C}_{W}=\frac{1}{2}(1+\tau)^{1 / \tau} C_{W}
$$

The modified width $\tilde{C}_{W}$ of the Gaussian wave packet is introduced to adjust the effect of density-dependent terms [24]. The Hamiltonian has 12 parameters shown in Table II. They are determined to reproduce the saturation properties of nuclear matter as well as ground state properties of finite nuclei.

In order to obtain the equilibrium configuration we adopt the QMD equations of motion with damping terms [24]:

$$
\begin{aligned}
\dot{\mathbf{R}}_{\mathbf{i}} & =\frac{\partial H}{\partial \mathbf{P}_{\mathbf{i}}}-\mu_{R} \frac{\partial H}{\partial \mathbf{R}_{\mathbf{i}}} \\
\dot{\mathbf{P}}_{\mathbf{i}} & =-\frac{\partial H}{\partial \mathbf{R}_{\mathbf{i}}}-\mu_{P} \frac{\partial H}{\partial \mathbf{P}_{\mathbf{i}}}
\end{aligned}
$$

where the damping coefficients $\mu_{R}$ and $\mu_{P}$ are positive definite and relate to the relaxation time scale.

As the QMD Hamiltonian used here contains momentum-dependent interactions ( $V_{\text {Pauli }}$ 
and $V_{\mathrm{MD}}$ ), we cannot use the usual expressions for the instantaneous temperature given as :

$$
\frac{3}{2} T=\frac{1}{\mathcal{N}} \sum_{i=1}^{\mathcal{N}} \frac{\mathbf{P}_{i}^{2}}{2 m_{i}}
$$

where $\mathcal{N}$ is the number of particles. Instead we use the effective temperature defined as [30]:

$$
\frac{3}{2} T_{\text {eff }}=\frac{1}{\mathcal{N}} \sum_{i=1}^{\mathcal{N}} \frac{1}{2} \mathbf{P}_{i} \cdot \frac{\partial \mathcal{H}}{\partial \mathbf{P}_{i}},
$$

which reduces to the usual definition of Eq. 15 if the Hamiltonian does not contain momentum-dependent interactions. Performing Metropolis Monte Carlo simulations it was shown in Ref. [25] that $T_{\text {eff }}$ is consistent with the temperature in the Boltzmann statistics.

In order to perform simulations at a specified temperature $\left(T_{\text {set }}\right)$ we adopt the NoséHoover thermostat [31 33] after suitably modifying it to adapt to the effective temperature [25]. The Hamiltonian including the thermostat is given by:

$$
\mathcal{H}_{\text {Nose }}=\sum_{i=1}^{\mathcal{N}} \frac{\mathbf{P}_{i}^{2}}{2 m_{i}}+\mathcal{U}\left(\left\{\mathbf{R}_{i}\right\},\left\{\mathbf{P}_{i}\right)\right\}+\frac{s^{2} p_{s}^{2}}{2}+g \frac{\ln s}{\beta}
$$

where $\left.\mathcal{U}\left(\left\{\mathbf{R}_{i}\right\}\right),\left\{\mathbf{P}_{i}\right\}\right)=\mathcal{H}-T$ is the potential depending on both positions and momenta, $s$ is the extended variable for the thermostat, $p_{s}$ is the momentum conjugate to $s, Q$ is the effective "mass" associated with $s$ taking a value $\sim 10^{8} \mathrm{MeV} \mathrm{fm}^{2}, g=3 \mathcal{N}$ needed to generate the canonical ensemble, and $\beta=1 / T_{\text {set }}$. The equations of motion for the extended system are written as:

$$
\begin{aligned}
\dot{\mathbf{R}}_{\mathbf{i}} & =\frac{\mathbf{P}_{i}}{m_{i}}+\frac{\partial \mathcal{U}}{\partial \mathbf{P}_{i}} \\
\dot{\mathbf{P}}_{\mathbf{i}} & =-\frac{\partial \mathcal{U}}{\partial \mathbf{R}_{\mathbf{i}}}-\xi \mathbf{P}_{i}, \\
\dot{\xi} & =\frac{1}{Q}\left[\sum_{i=1}^{\mathcal{N}}\left(\frac{\mathbf{P}_{i}}{m_{i}}+\mathbf{P}_{i} \cdot \frac{\partial \mathcal{U}}{\partial \mathbf{P}_{i}}\right)-\frac{g}{\beta}\right] \\
\dot{s} / s & =\xi
\end{aligned}
$$

where $\xi\left(=s p_{s} / Q\right)$ acts as thermodynamic friction coefficient. When the system is evolved according to the above equations $\mathcal{H}_{\text {Nose }}$ remains conserved and $T_{\text {eff }}$ fluctuates around $T_{\text {set }}$.

\section{SIMULATION}

Adopting the theoretical framework outlined in the previous section we perform QMD simulations of nuclear matter for a wide range of density $\left(\rho=0.1-0.775 \rho_{0}\right)$ and temperature 
$(T=1-9 \mathrm{MeV})$ relevant for the study of the liquid-gas phase transition. We investigate symmetric nuclear matter (proton fraction $Y_{p}=0.5$ ) important for heavy-ion collisions as well as asymmetric nuclear matter with $Y_{p}=0.3$, typical for supernova environment and $Y_{p}=0.1$, relevant for neutron stars. We take into account 2048 nucleons for $Y_{p}=0.5$ and 0.3 , and 16384 nucleons for $Y_{p}=0.1$ in a cubic box the size of which is determined from the number of particles and the chosen density. Periodic boundary conditions are imposed to simulate infinite matter. The number of protons (neutrons) with spin-up is taken to be equal to that of protons (neutrons) with spin-down. To calculate the Coulomb interaction we employ the Ewald method [26, 33], where electrons are considered to form a uniform background and make the system charge neutral. To study the nuclear matter at sub-saturation densities several authors [34 36] have considered the Coulomb interaction as a Yukawa-type interaction where the choice of screening length $(\lambda)$ is not very well defined. However, in a recent study [37] it was shown that the the results may depend on $\lambda$, significantly. The Ewald method used here does not suffer from this shortcoming.

As an initial configuration we distribute nucleons randomly in phase space. Then with the help of the Nosé-Hoover thermostat we equilibrate the system at $T \sim 20 \mathrm{MeV}$ for about $2000 \mathrm{fm} / \mathrm{c}$. To achieve the ground state configuration we then slowly cool down the system in accordance with the damped equations of motion (Eqs. 14) until the temperature reaches a value below $1 \mathrm{keV}$. In order to obtain nuclear matter configuration at a finite temperature $T_{\text {set }}$ we cool down the system until $T$ reaches $\sim 5 \mathrm{MeV}$. Then the system is relaxed for 5000

$\mathrm{fm} / \mathrm{c}$ at the desired temperature $T_{\text {set }}$ with the help of the thermostat and finally, it is further relaxed without the thermostat for another $5000 \mathrm{fm} / \mathrm{c}$. All the measurements are taken at this last stage of simulation.

\section{RESULTS}

In Fig. 1 we show simulation snapshots for the nucleon distributions of asymmetric matter with $Y_{p}=0.3$, at $\rho=0.1 \rho_{0}$ and $T=0$. The snapshot in the left (right) panel is obtained when the Coulomb interaction is (not) taken into account. As expected, we get a single large cluster with several dripped neutrons in absence of the repulsive Coulomb interactions. On the other hand, in presence of the Coulomb interaction we observe several smaller clusters arranged in a lattice. 

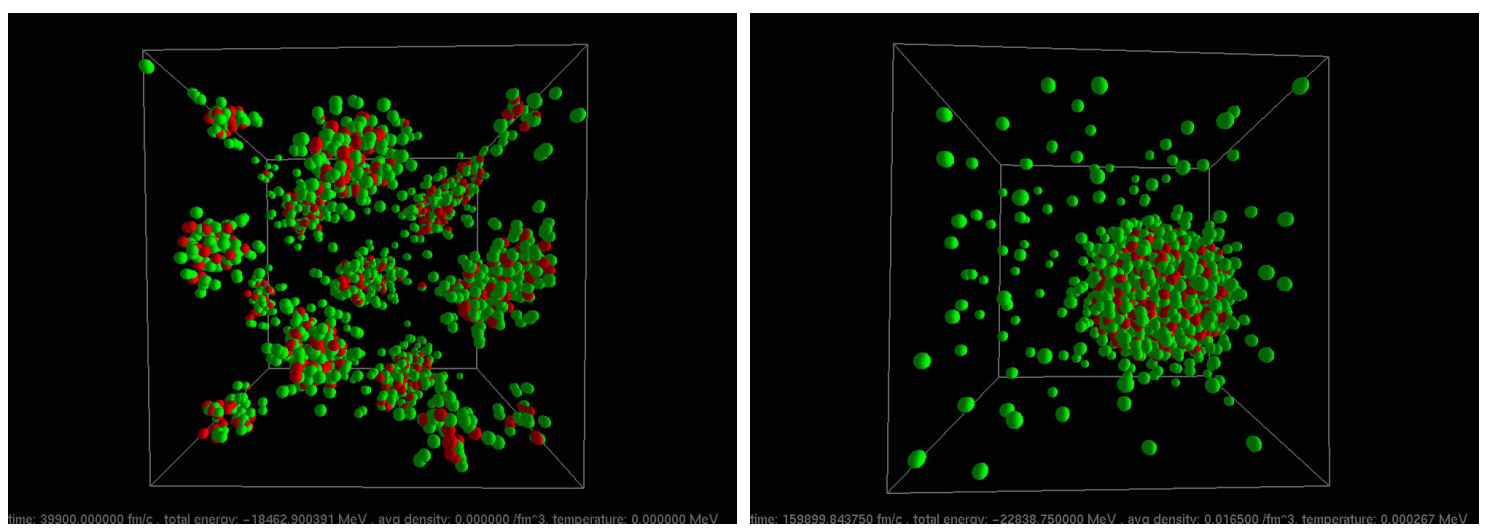

FIG. 1. Snapshots from simulations showing distribution of nucleons at $0.1 \rho_{0}, Y_{p}=0.3, T=0$ with (left) and without (right) the Coulomb interaction. Green (red) spheres represent neutrons (protons).

For the analysis of nucleon distribution in space we calculate the two-point correlation function $\xi_{i i}$ for the nucleon density fluctuation defined as [26, 29]:

$$
\triangle^{(i)}=\frac{\rho^{(i)}(\mathbf{x})-\rho_{\mathrm{av}}^{(i)}}{\rho_{\mathrm{av}}^{(i)}}
$$

where $i=n, p, N$ denotes neutrons, protons and nucleons, respectively and $\rho_{\mathrm{av}}^{(i)}=\mathcal{N}^{(i)} / V$. Then the correlation function is given by

$$
\xi_{i i}(r)=\left\langle\triangle_{i}(\mathbf{x}) \triangle_{i}(\mathbf{x}+\mathbf{r})\right\rangle
$$

where the average is taken over the position $\mathbf{x}$ and the direction of $\mathbf{r}$.

\section{A. Symmetric nuclear matter}

To investigate the role of the Coulomb interaction on the liquid-gas phase transition of symmetric nuclear matter we perform simulations for the whole range of density and temperature mentioned earlier with and without Coulomb interaction. We take 2048 nucleons and obtain the ground state configurations following the procedure described in the previous section. The phase transition region is determined by calculating the two-point correlation functions. In Fig. 2 we plot the two-point correlation functions for nucleons around the phase transition density at $T=0$, with (left panel) and without (right panel) Coulomb interaction. It is clear from the figure that the long-range correlation of the nucleon distribution vanishes between $0.7-0.725 \rho_{0}$ when Coulomb interaction is considered. This value 

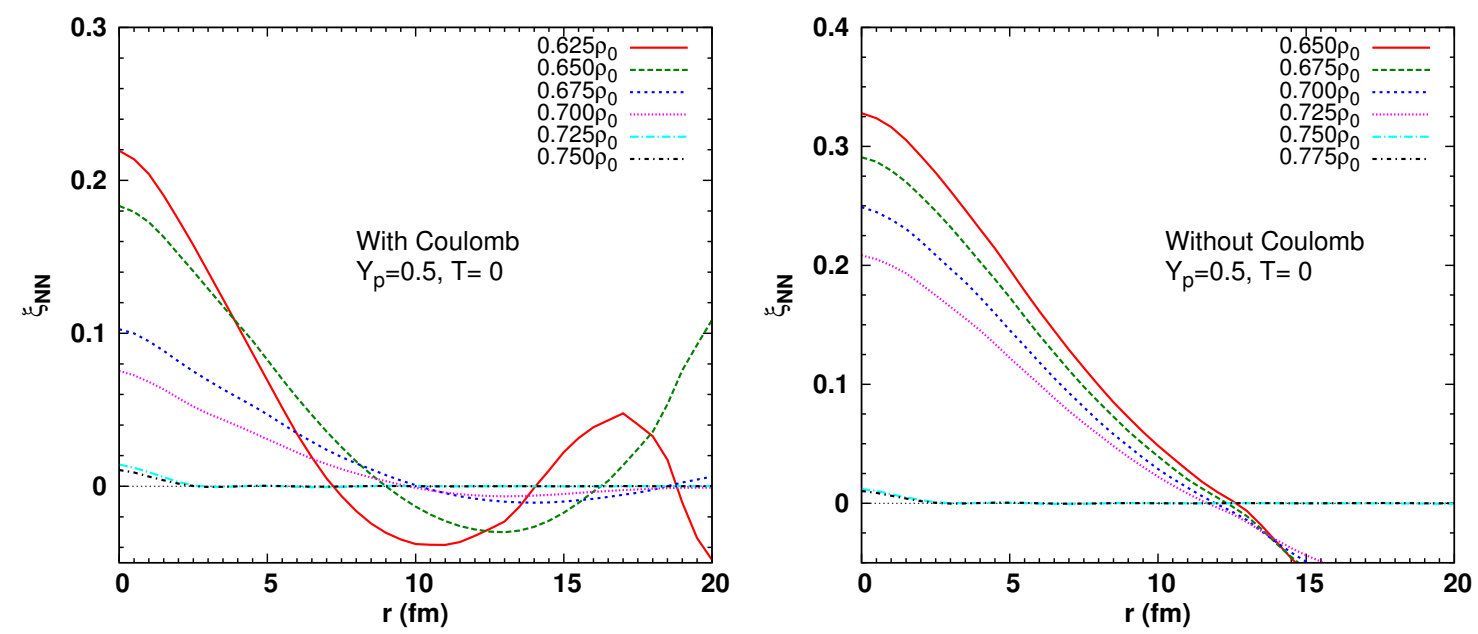

FIG. 2. Two-point correlation function $\xi_{N N}$ around the liquid-gas phase transition region at $T=0$ with (left panel) and without (right panel) Coulomb interaction for symmetric nuclear matter.

is in agreement with that of an earlier calculation [26] with the same model. On the other hand, if the Coulomb interaction is not considered the long-range correlation disappears between $0.725-0.75 \rho_{0}$. In other words, the Coulomb interaction shifts the transition from the liquid phase to the gas phase to lower density.

In Fig. 3 the two-point correlation function $\xi_{N N}$ is shown for the cases with (left panel) and without (right panel) Coulomb interaction at $\rho=0.4 \rho_{0}$, as a typical example. The figures in the bottom panel are the zoomed version of the corresponding figures in the top panel. From the figures we find that although with increasing temperature the amplitude of $\xi_{N N}$ decreases, its first zero-point that corresponds to the size of the clusters doesn't change much. This behaviour was also seen in earlier calculations [25, 26]. However, in absence of repulsive Coulomb interaction between protons, nucleons are expected to form larger clusters. This is exactly seen here as the first zero in $\xi_{N N}$ is reached at larger values of $r$ when the Coulomb interaction is not considered. A Interestingly, one can also observe that the disappearance of long-range correlations that marks the transition from inhomogeneous to homogeneous matter takes place between $T=4$ and $5 \mathrm{MeV}$ in presence of the Coulomb interaction and between $T=6$ and $7 \mathrm{MeV}$ without Coulomb interaction.

Following the same procedure we determine the phase transition temperatures for the whole range of densities considered here for both with and without Coulomb interaction. Accumulating all the results we obtain the phase diagrams shown in Fig. 4. Comparing the results of two cases we can see that the phase transition temperature is always larger by 

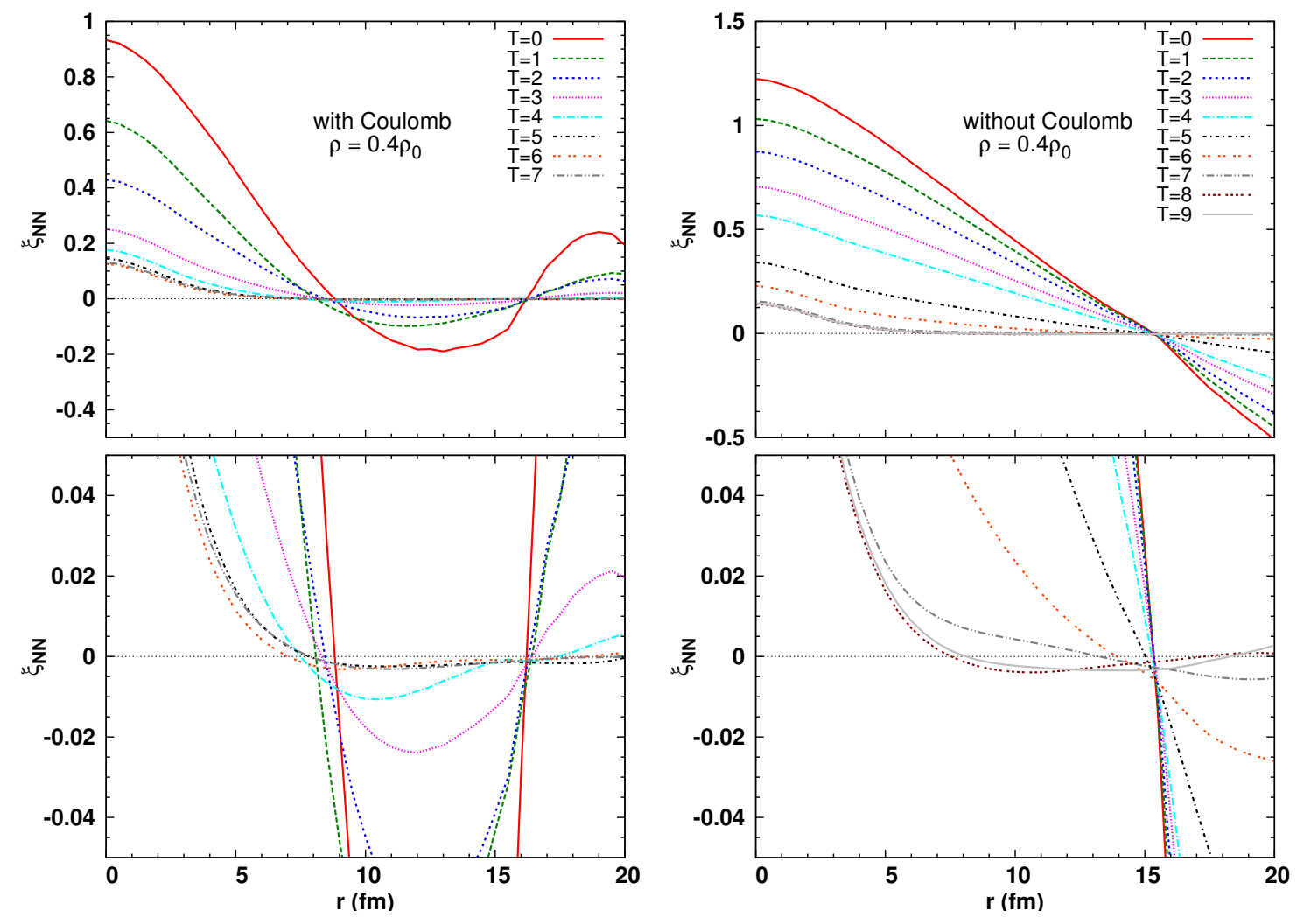

FIG. 3. Two-point correlation function $\xi_{N N}$ at $\rho=0.4 \rho_{0}$ with (left panel) and without (right panel) Coulomb interaction for symmetric nuclear matter. The figures in the bottom panel are zoomed versions of the corresponding figures in the top panel
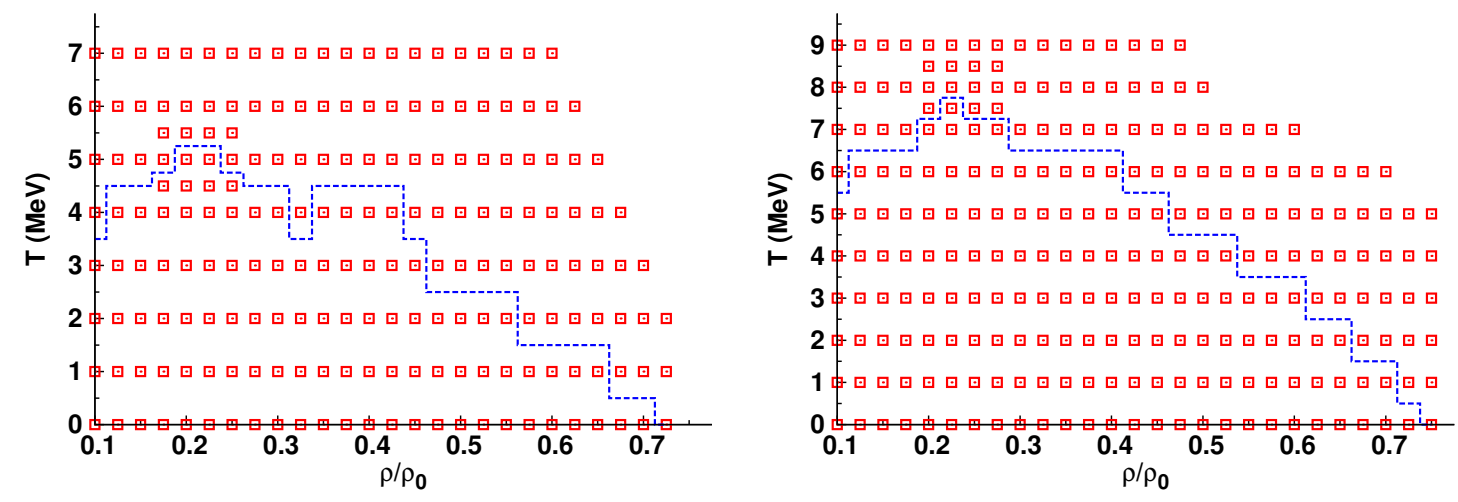

FIG. 4. Phase diagram for symmetric nuclear matter with (left panel) and without (right panel) Coulomb interaction. The dashed line indicates the phase transition line. 

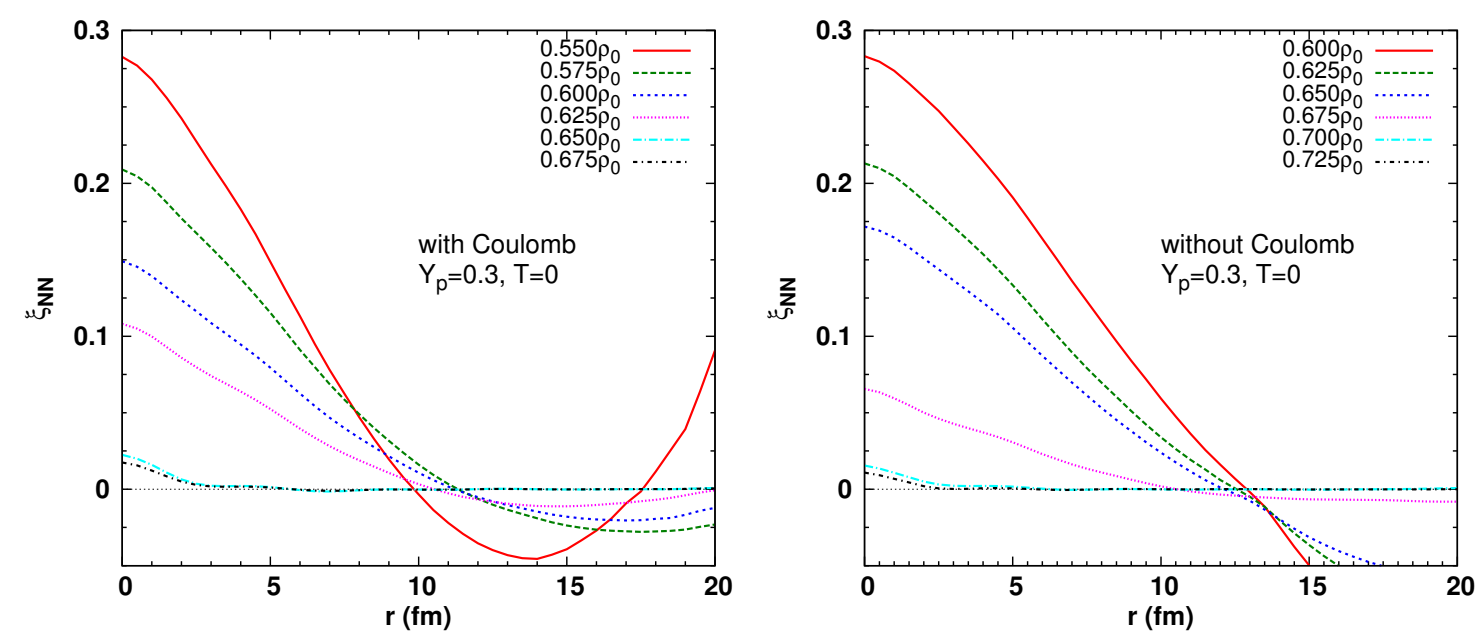

FIG. 5. Same as Fig. 2 but for asymmetric nuclear matter with $Y_{p}=0.3$

$\gtrsim 1-2 \mathrm{MeV}$ for the case without Coulomb interaction (right panel) than when including Coulomb (left panel). From the phase diagrams it can also be observed that in the Coulomb case the critical end point of the liquid-gas phase transition is located at $\rho_{c} \simeq 0.225-0.25 \rho_{0}$ and $T_{c} \gtrsim 5 \mathrm{MeV}$. This value is similar to the result obtained in an earlier calculation with the same QMD Hamiltonian [25]. On the other hand, the critical point is located at $\rho_{c} \sim 0.225$ and $T_{c} \gtrsim 7.5 \mathrm{MeV}$ when Coulomb interaction is not taken into consideration. The important point to note is that the Coulomb interaction reduces the critical temperature $T_{c}$ by $\sim 2 \mathrm{MeV}$ but the critical density $\rho_{c}$ remains unchanged. This behaviour was also seen in the calculation of Jaqaman et al [9] for symmetric nuclear matter described by Skyrme interactions.

\section{B. Asymmetric nuclear matter with $Y_{p}=0.3$}

Next, we investigate the liquid-gas phase transition region for asymmetric nuclear matter with $Y_{p}=0.3$, a value typical for supernova environments. We take 2048 nucleons (608 protons and 1440 neutrons) for this calculation. In this case also we calculate the two-point correlation functions to determine the phase boundary of the liquid-gas transition. In Fig. 5. we plot the correlation function $\xi_{N N}$ at densities close to the phase transition region for nuclear matter with $Y_{p}=0.3$ and $T=0$, with (left panel) and without (right panel) Coulomb interaction. As in the case of symmetric matter here we also find that the Coulomb interaction decreases the liquid-gas transition density. With the Coulomb interaction the 

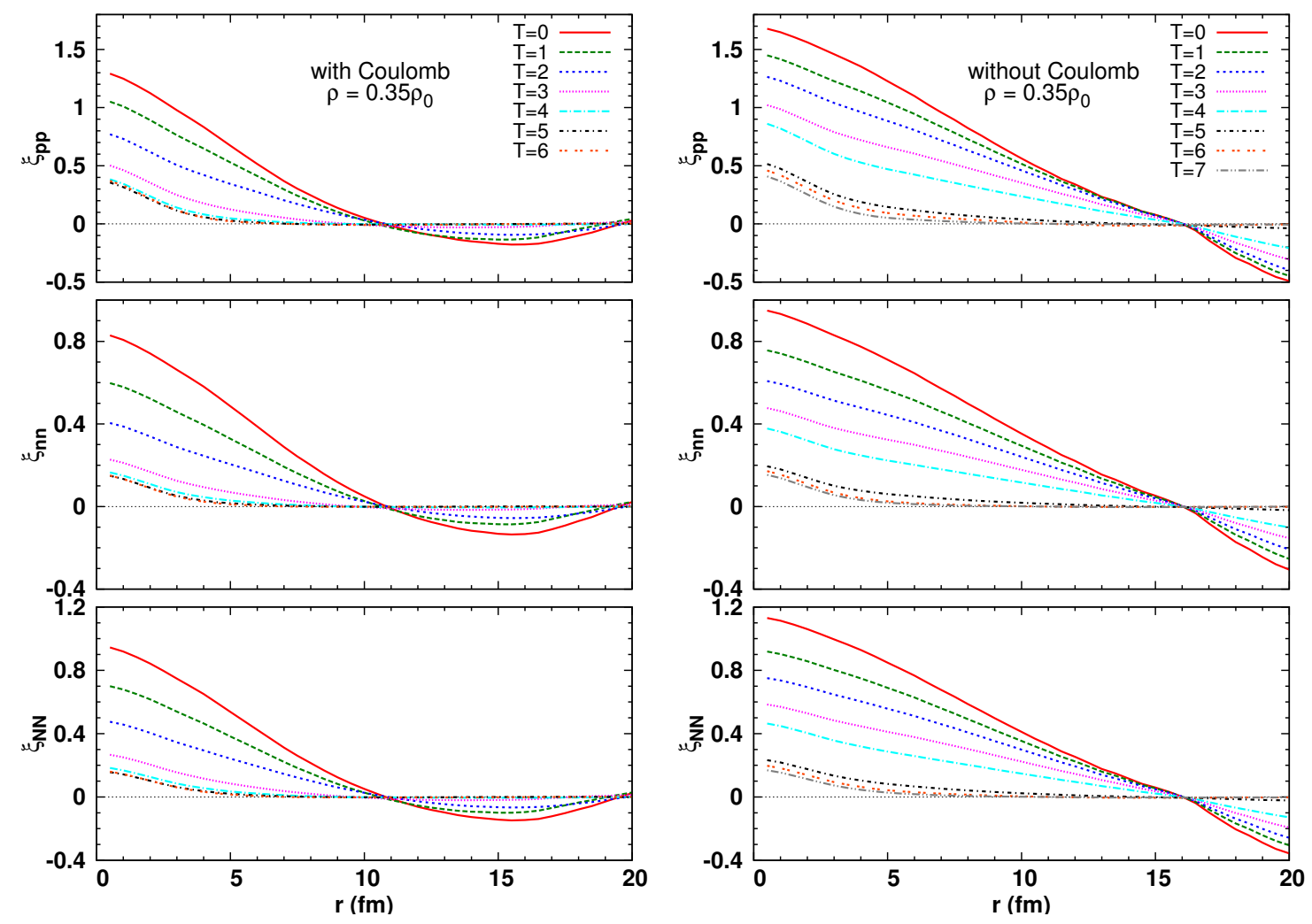

FIG. 6. Two-point correlation functions at $\rho=0.35 \rho_{0}$ with (left panel) and without (right panel) Coulomb interaction for asymmetric nuclear matter with $Y_{p}=0.3$.

transition happens within the density range between 0.625 and $0.65 \rho_{0}$ (reported earlier in Ref. [29]), whereas without Coulomb interaction the same happens between 0.675 and $0.7 \rho_{0}$.

We plot the two-point correlation functions $\xi_{p p}, \xi_{n n}$ and $\xi_{N N}$ with and without considering the Coulomb interaction at a typical example density $\rho=0.35 \rho_{0}$, in Fig. 6. The amplitudes of $\xi_{n n}$ are found to be lower than those of $\xi_{p p}$ due to the presence of uniformly distributed dripped neutrons. The higher amplitudes of $\xi_{i i}$ in absence of the Coulomb interaction point to the fact that the particles are more clustered in this case as is also seen in Fig. 1. Likewise in symmetric matter here also the first zero-point of all $\xi_{i i}$ does not change much with temperature. Moreover, the first zero-point of $\xi_{p p}$ coincide with the one of $\xi_{n n}$ for all temperatures showing a strong correlation between the density fluctuations of neutrons and protons even at $Y_{p}=0.3$. From the figure we similarly find that the phase transition line lies between $T=3-4 \mathrm{MeV}$ and $T=5-6 \mathrm{MeV}$ for the cases with and without Coulomb interaction, respectively.

We continue the determination of the phase transition line for all other densities and 

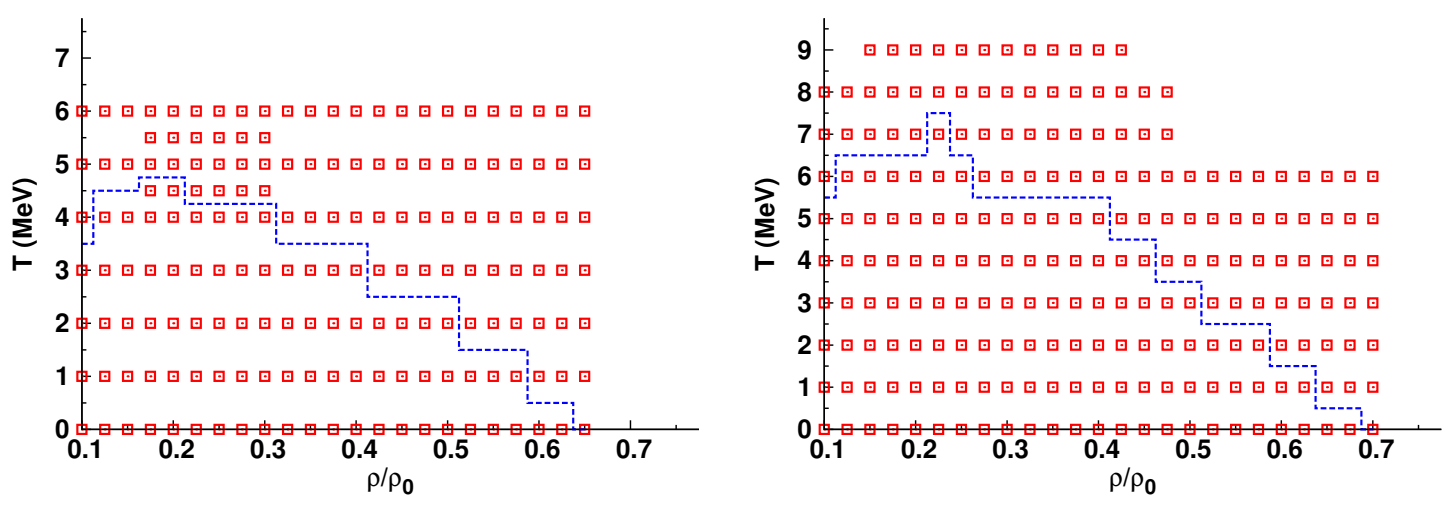

FIG. 7. Same as Fig. 4, for $Y_{p}=0.3$

obtain the phase diagram shown in Fig. 7. When we do not consider the Coulomb interaction the transition temperature is always higher by $\gtrsim 1-2 \mathrm{MeV}$ compared to the case including Coulomb interaction, analogously to the results for symmetric matter. The critical point of the transition is located at $T_{c} \gtrsim 7 \mathrm{MeV}, \rho_{c} \sim 0.225 \rho_{0}$ without the Coulomb interaction and at $T_{c} \gtrsim 4.5, \rho_{c} \sim 0.2 \rho_{0}$ with Coulomb. As for $Y_{p}=0.5$ the critical density is not much affected by the Coulomb interaction but the critical temperature is decreased by $\gtrsim 3 \mathrm{MeV}$. A comparison of the results for $Y_{p}=0.5$ and 0.3 reveals that the critical point is similar in absence of Coulomb interactions. However, including Coulomb the critical point gets shifted slightly to lower density and temperature.

\section{Asymmetric nuclear matter with $Y_{p}=0.1$}

Finally, we investigate the liquid-gas phase transition with and without Coulomb interaction for even more asymmetric nuclear matter with $Y_{p}=0.1$ which is close to values relevant for the neutron star interior. In order to have enough number of protons that play a crucial role in generating long-range correlations we use 16384 nucleons (1600 protons and 14784 neutrons) in our simulations for this case. The ground state configurations are obtained following the same procedure as earlier. To determine the phase boundary of liquid-gas transition we again calculate two-point correlation functions. In Fig. 8, we plot the correlation function $\xi_{N N}$ at densities close to the phase transition region for nuclear matter with $Y_{p}=0.1$ and $T=0$, with (left panel) and without (right panel) Coulomb interaction. Unlike the cases of $Y_{p}=0.5$ and 0.3 here we find that the Coulomb interaction slightly increases 

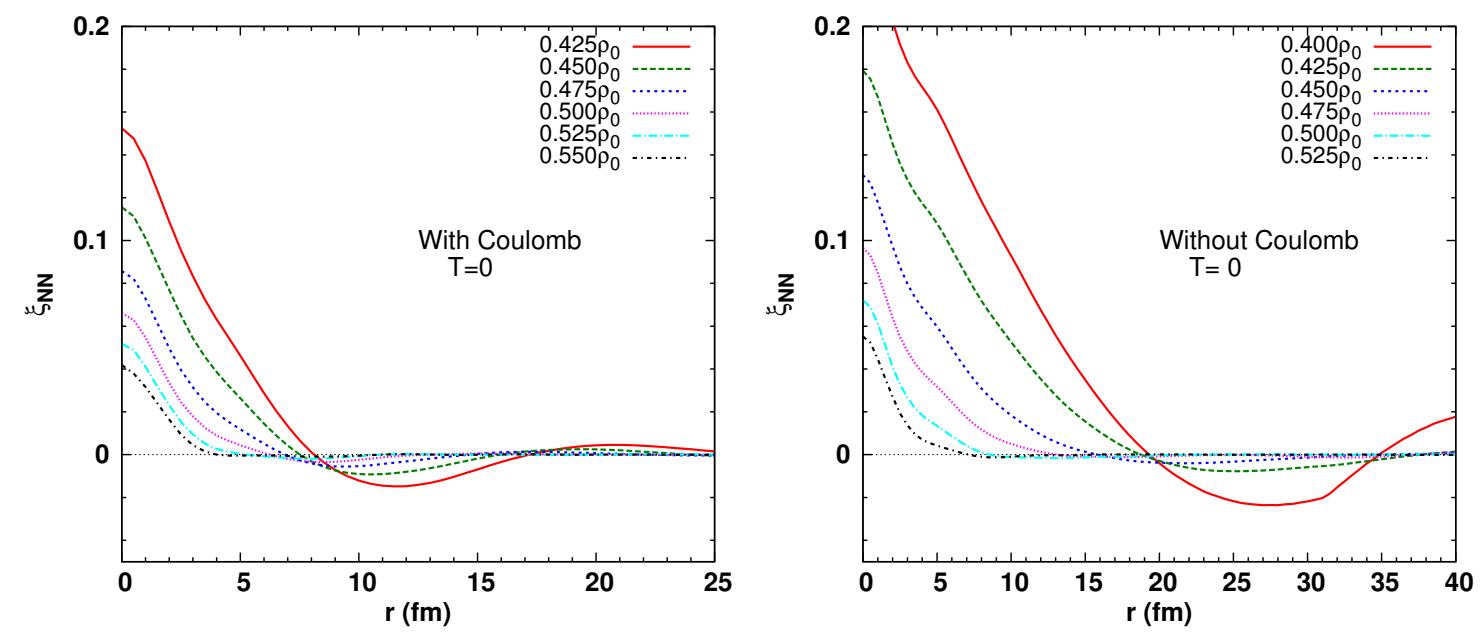

FIG. 8. Two-point correlation as in Fig. 5 for $Y_{p}=0.1$.
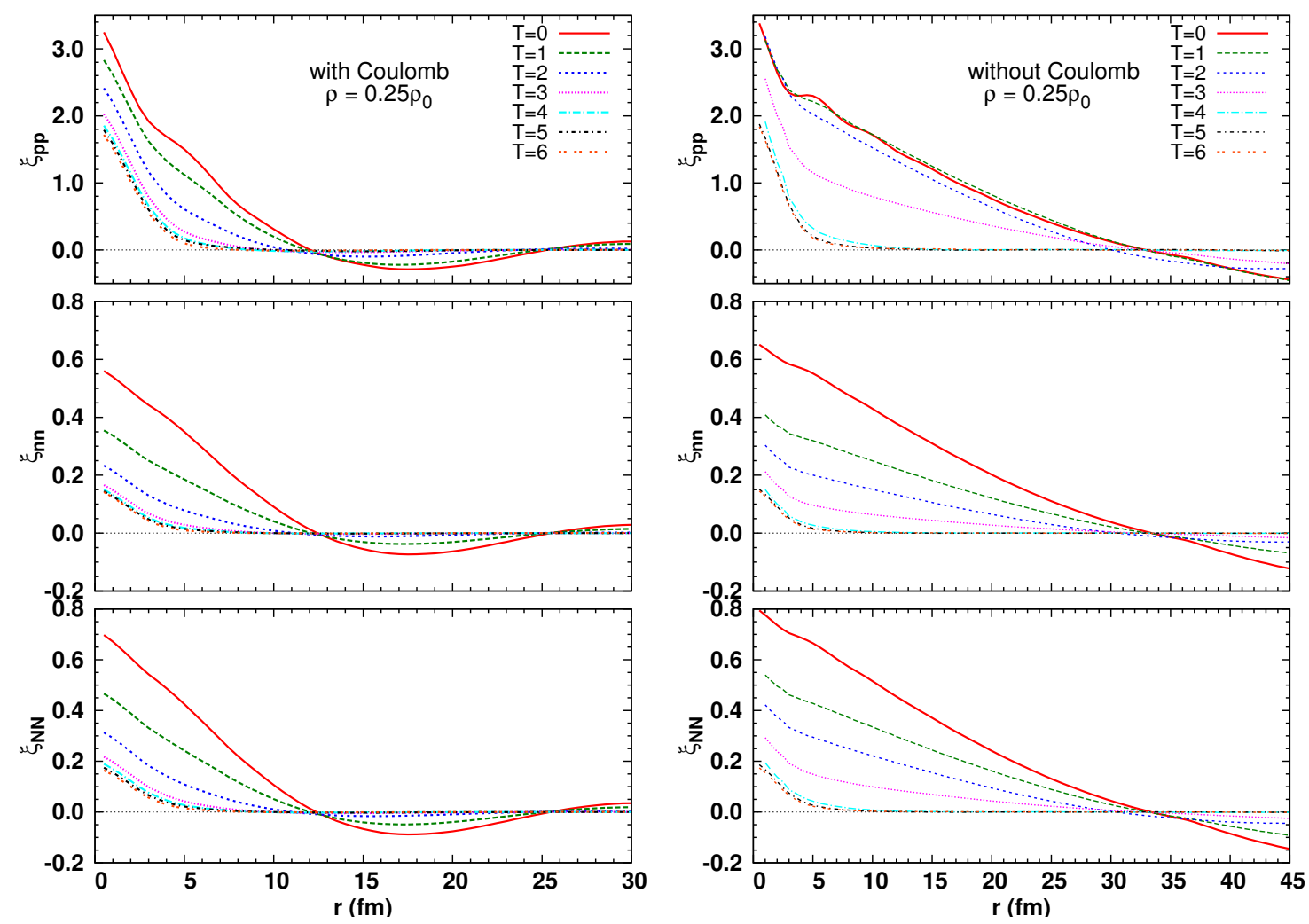

FIG. 9. Two-point correlation functions at $\rho=0.25 \rho_{0}$ with (left panel) and without (right panel) Coulomb interaction for asymmetric nuclear matter for $Y_{p}=0.1$.

the transition density. Including Coulomb interactions the transition happens at a density range between 0.475 and $0.5 \rho_{0}$ whereas, without the Coulomb interaction this happens to be in the interval from 0.45 to $0.475 \rho_{0}$. 

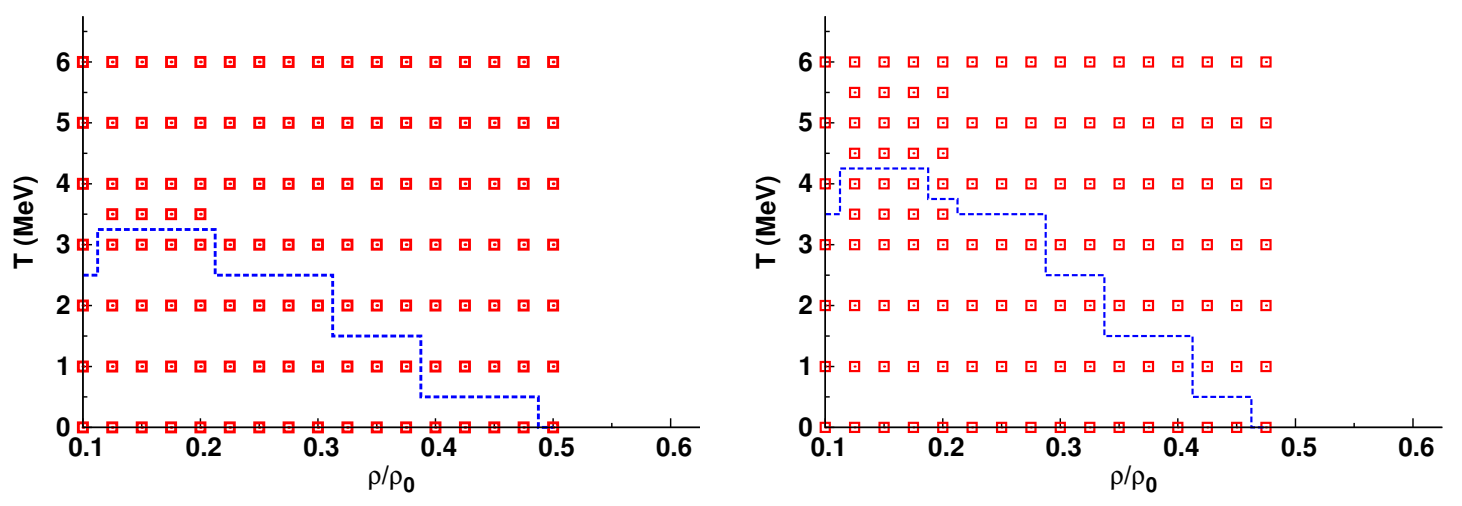

FIG. 10. Phase diagrams as in Fig. 2, but for $Y_{p}=0.1$.

Next, we plot different two-point correlation functions with and without considering the Coulomb interaction at a typical example density $\rho=0.25 \rho_{0}$, in Fig. 9. The difference between $\xi_{p p}$ and $\xi_{n n}$ is even higher than that for $Y_{p}=0.3$, because the number of dripped neutrons also increases with decreasing $Y_{p}$. Even in this highly asymmetric matter neutrons and protons are found to be highly correlated as the locations of first zero-point of $\xi_{i i}$ coincide. It is also seen from the figure that the long-range correlation vanishes between $T=2-3 \mathrm{MeV}$ and $T=3-4 \mathrm{MeV}$ for the cases with and without the Coulomb interaction, respectively.

After determining the phase transition line for all other densities we obtain the phase diagram shown in Fig. 10. From the figure we can see that the phase transition temperatures for the two cases differ by $T \sim 1 \mathrm{MeV}$ at low densities $\left(<0.3 \rho_{0}\right)$. However, at higher densities the difference vanishes. The critical point of the transition is located at $T_{c} \gtrsim 4$ $\mathrm{MeV}, \rho_{c} \sim 0.125 \rho_{0}$ without the Coulomb interaction and at $T_{c} \gtrsim 3, \rho_{c} \sim 0.125 \rho_{0}$ with the Coulomb interaction. As in the cases of $Y_{p}=0.5$ and 0.3 the critical density is not much affected by the Coulomb interaction but the critical temperature is decreased by only $\sim 1$ $\mathrm{MeV}$ in this case. If the results of $Y_{p}=0.3$ and 0.1 are compared one can observe that the shift in critical point is larger when the Coulomb interaction is not considered.

To investigate whether the reduction of the critical temperature in presence of the Coulomb interaction depends on the nuclear force, especially on the surface energy we also perform simulations with another QMD model [30] that includes a surface term . In this case we also found that the Coulomb interaction reduces the critical temperature whereas the 
critical density remains largely unchanged. For this model, the critical point of the liquid-gas phase transition of nuclear matter with $Y_{p}=0.3$ is given by $T_{c}=10 \mathrm{MeV}, \rho_{c} \sim 0.25 \rho_{0}$, without Coulomb and by $T_{c}=8 \mathrm{MeV}, \rho_{c} \sim 0.225 \rho_{0}$, with Coulomb. The values of the critical temperature and density found here in presence of the Coulomb interaction are very similar to the values obtained by Sonoda et al [38] in an earlier study. With increasing density and/or temperature the surface energy that depends on the gradient of density across the surface, becomes smaller. Therefore, the reduction in critical temperature happens mainly due to the Coulomb energy and holds for all nuclear models.

\section{SUMMARY AND CONCLUSION}

We have investigated the effect of the Coulomb interaction on the liquid-gas phase transition of nuclear matter using molecular dynamics simulations. We have performed simulations for a wide range of density and temperature with and without Coulomb interaction for this purpose. We have considered both symmetric nuclear matter, relevant for heavy-ion physics as well as asymmetric matter with $Y_{p}=0.3$ and 0.1 , important for supernova and neutron star matter, respectively. To determine the phase transition region we have calculated the two-point correlation functions of the fluctuations of nucleon densities. The temperatures at which the transition from the liquid phase to the gas phase take place at various densities are obtained by determining the location where the long-range correlations vanish. We also determine the critical point of the liquid-gas phase transition of nuclear matter for all three $Y_{p}$ s considered here. We found that although the Coulomb interaction lowers the critical temperature by $\sim 2-3 \mathrm{MeV}$ for nuclear matter with $Y_{p}=0.5$ and 0.3 and by $\sim 1 \mathrm{MeV}$ for $Y_{p}=0.1$, the critical density remains more or less unchanged. It could also be observed that the the densities at which the liquid-gas transition takes place at $T=0$, is higher if the Coulomb interaction is not considered for the cases of $Y_{p}=0.5$ and 0.3 . However, for $Y_{p}=0.1$, there is not much difference in the transition density. For this highly asymmetric matter the difference between the phase diagrams with and without Coulomb is much smaller than for the other two values of $Y_{p}$. This is the case because the Coulomb energy becomes less important for highly asymmetric matter. We also showed that the main conclusion that the Coulomb interaction reduces the critical temperature but the critical density remain unchanged, is independent of nuclear model specifics. 
Based on these findings we plan to investigate susceptibilities of particle numbers around the phase transition line and critical end-point, as such studies are directly related to the more general search for observable signals of structures in the phase diagram of strongly interacting matter comparing to observables from heavy-ion collisions.

[1] M. D'Augostino et al, Nucl. Phys. A 749, 55 (2005).

[2] C. B. Das, S. Das Gupts, W. G. Lynch, A. Z. Mekjian, and M. B. Tsang, Phys. Rep. 406, 1 (2005).

[3] M. Hempel and J. Schaffner-Bielich, Nucl. Phys. A 837, 210 (2010).

[4] H. Pais, S. Chiacchiera and C. Providência, Phys. Rev. C 91, 055801 (2015)

[5] C. Ducoin, K. H. O. Hasnaoui, P. Napolitani, Ph. Chomaz, and F. Gulminelli, Phys. Rev. C 75, 065805 (2007).

[6] S. Typel, H. H. Wolter, G. Röpke, and D. Blaschke, Eur. Phys. J. A 50, 17 (2014)

[7] B. K. Sharma and S. Pal, Phys. Rev. C 82, 055802 (2010).

[8] A. R. Raduta, and F. Gulminelli, Phys. Rev. C 82, 065801 (2010)

[9] H. R. Jaqaman, A. Z. Mekjian, and L. Zamick, Phys. Rev. C 27, 2782 (1983); 29, 2067 (1984).

[10] J. M. Lattimer, C. J. Pethick, D. G. Ravenhall, and D. Q. Lamb, Nucl. Phys. A 432, 646 (1985).

[11] C.J. Pethick, D.G. Ravenhall, and C.P. Lorenz, Nucl. Phys. A 584, 675 (1995).

[12] V. M. Kolomietz, A. I. Sanzhur, S. Shlomo, and S. A. Firin 64024315 (2001).

[13] C. Ducoin, Ph. Chomaz and F. Gulminelli, Nucl. Phys. A 77168 (2006).

[14] H. Müller, and B. D. Serot, Phys. Rev. C 52, 2072 (1995).

[15] P. Wang, Phys. Rev. C. 61, 054904 (2000).

[16] S. S. Avancini, L. Brito, Ph. Chomaz, D. P. Menezes, and C. Providência, Phys. Rev. C 74, 024317 (2006)

[17] C. Wellenhofer, J. W. Holt, and N. Kaiser, Phys. Rev. C 92, 015801 (2015).

[18] M. Hempel, V. Dexheimer, S. Schramm and I. Iosilevskiy, Phys. ReV. C 88, 014906 (2013).

[19] S. J. Lee and A. Z. Mekjian, Phys. Rev. C 63, 044605 (2001).

[20] T. Sil, S. K. Samaddar, J. N. De, and S. Shlomo, Phys. Rev. C 69, 014602 (2004) .

[21] S. S. Avancini, D. P. Menezes, M. D. Alloy, J. R. Marinelli, M. M. W. Moraes, and C. 
Providência, Phys. Rev. C 78, 015802 (2008).

[22] S. S. Bao and H. Shen, Phys. Rev. C 89, 045807 (2014).

[23] S. S. Bao and H. Shen, Phys. Rev. C 93, 025807 (2016).

[24] T. Maruyama, K. Niita, K. Oyamatsu, T. Maruyama, S. Chiba and A. Iwamoto, Phys. Rev. C 57, 655 (1998).

[25] G. Watanabe, K. Sato, K. Yasuoka and T. Ebisuzaki, Phys. Rev. C 69, 055805 (2004).

[26] G. Watanabe, K. Sato, K. Yasuoka and T. Ebisuzaki, Phys. Rev. C 68, 035806 (2003).

[27] G. Watanabe, T. Maruyama, K. Sato, K. Yasuoka and T. Ebisuzaki, Phys. Rev. Lett. 94, $031101(2005)$

[28] G. Watanabe, H. Sonoda, T. Maruyama, K. Sato, K. Yasuoka and T. Ebisuzaki, Phys. Rev. Lett. 103, $121101(2009)$

[29] R. Nandi and S. Schramm, Phys. Rev. C 94, 025806 (2016).

[30] S. Chikazumi, T. Maruyama, S. Chiba, K. Niita, and A. Iwamoto, Phys. Rev. C 63, 024602 (2001).

[31] S. Nosé, J. Chem. Phys. 81, 511 (1984).

[32] W. G. Hoover, Phys. Rev. A 31, 1695 (1985).

[33] M. P. Allen and D. J. Tildesley, Computer Simulation of Liquids (Clarendon, Oxford, 1987)

[34] C. J. Horowitz, Pérez-García and J. Piekarwicz, Phys. Rev. C 69, 045804 (2004).

[35] A. S. Schneider, c. J. Horowitz, J. Hughto and D. K. Berry, Phys. Rev. C 88, 065807 (2013).

[36] C. O. Dorso, P. A. Giménez Molinelli and J. A. López, Phys. Rev. C 86, 055805 (2012).

[37] P. N. Alcain, P. A. Giménez Molinelli, J. I. Nichols and C. O. Dorso, Phys. Rev. C 89, 055801 (2014).

[38] H. Sonoda, G. Watanabe, K. Sato, K. Yasuoka and T. Ebisuzaki, Phys. Rev. C 77, 035806 (2008). 